\title{
A Design of Context aware Smart Home Safety Management using by Networked RFID and Sensor
}

Byunggil Lee and Howon Kim,

Electronics and Telecommunications Research Institute, Korea

\begin{abstract}
Recently home intelligent service is extended to context aware and situation aware automation service by using ubiquitous technology. In this paper, we focused on user's context based intelligent security management for home safety application using RFID and WSN(Wireless Sensor Network). We propose a context aware home safety application model. The proposed context service support security and privacy control by using user's situation aware security management. It will be a practical application of RFID and sensor network for ubiquitous home safety environment. The proposed system has lots of advantages in user safety and energy efficiency.
\end{abstract}

\section{System Architecture}

Home intelligent services may be generally defined with home network service, home automation service, home theater service, and internet service to interwork other device or networks in inside and outside of home. Recently home intelligent service is extended to context aware and situation aware automation service by using ubiquitous technology. Ubiquitous services will be available to support the user identification and situation awareness with interconnected various sensors and user with RFID tags.

As an ubiquitous technology, radio frequency identification(RFID) has proven to be a powerful solution for not only supply chain management (SCM), inventory management and businesses process improvement [1], [2] but also access control and safety. The RFID is an automatic identification technology whereby digital data encoded in an RFID tag is captured by reader using RF signal. It is different

Please use the following format when citing this chapter:

I.ee, B., Kim, H., 2007, in IFIP International Federation for Information Processing. Volume 256, Home Networking. $\mathrm{AI}$ Agha, K., Carcelle, X.. Pujolle, G., (Boston: Springer), pp. 215-224. 
from bar code in usage of tag memory, identity multi-items, writing of new data, interfacing with sensor and digital data source.

Using RFID and sensor network, we can store all information about the reading item in a networked information server with backend database, and ensure reliable and timely update context information based on physical user's location at home.

RFID systems are increasingly being used in high security applications, such as access systems for making payments or issuing tickets. However, the use of RFID and sensor network in these applications necessitates the use of security measures to protect against attempted attacks, in which people try to trick the RFID and sensor related system in order to gain unauthorized access to home network.

On the other hand, home network has various threats, include cyber attacks of Internet, security homes against hacking, malicious codes, worms, viruses, DoS attacks, and eavesdropping, since it is connected to the access network.

In this paper, RFID and sensor technology is applied surveillance and detection of intrusion in home security systems. Using these kinds of management systems, an innovative business process can be constructed and various convenient services can be realized [3]. However, unless these security systems are situation based designed and context based constructed, they can cause severe problem to home user when user be in trouble or have an accident by same security rule [4].

So, we proposed a context aware RFID system which has been integrated to identification RFID Tag and sensor network for acquisition time and location of context. Context aware based RFID means that equipment is able to take actions automatically on capturing event time and location of user Tag. The proposed context aware RFID system is capable of recognizing such a situation and determining the use of the resource based on the recognized location and time situation information. The primary purpose of this paper is to design of context aware RFID system and sensor network. The secondary purpose of this paper is to analyze about the access control and security management for home networking application. The remainder of this paper is organized as follows, Section 2 gives a brief description of intelligent home safety service architecture. Section 3 delineates a model of context based home security management service by using RFID and sensor network. Finally, conclusions and a discussion of advantages are presented in Section 4. 


\section{RFID and Sensor Network based Ambient Home Service Architecture}

Many companies and organizations, including EPC Global Network[5], have developed RFID systems and international standardization is ongoing. The basic architecture of an RFID system is shown in Fig. 1.

For user's specific location based RFID service, we also include a mobile terminal with RFID reader which is read simply RFID tag for identify the objects. Users get information of objects by reading RFID tag and resolve it by inquiring to a network through mobile network. In the platform, WIPI(A kind of Korea's mobile standard platform. It is based on Java platform) is used as a RFID platform.

Typical applications will be a B2C model such as after sale service in home appliance or authentication service of a stuff(pearl, portrait) or guide to a demo video download and advance booking triggered by a mobile phone's menu after read RFID code from tag attached movie poster.

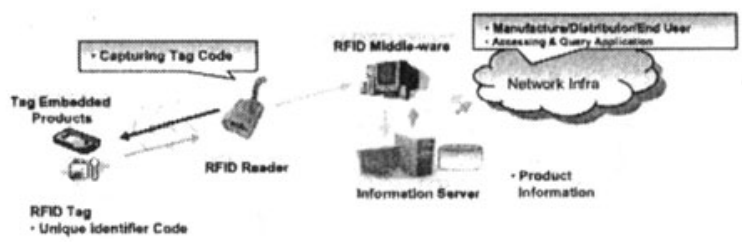

Figure 1. The basic architecture of RFID system.

As shown in Figure 1, quite briefly, RFID systems consist of RFID tag embedded product, Reader, RFID middle-ware, ONS (Object Name Service) for IS lookup, IS(Information Server) for product business data with code and the accessing application[5].

The context based RFID and sensor network is suitable for the coming ubiquitous environment, and situation information for the sensors and RFID identification Tag[7][8].

In the near future, Tag component of RFID and sensor will be integrated to sensing Tag. Home intelligent systems provide automatic home device control of the conditions of indoor environments by context based control service[10].

In this paper, however, we focus on the automated processing of user's context related safety and control functions, such as gate control, parking-gate control, room light control, room temperate control and device control such as TV, audio, 
window, curtain and safety level in home security system when user fall asleep or absent.

In our system, called " $u$-IHS(Ubiquitous computing based Intelligent Home Safety management service)", we use the RFID technology for context based identifying the user, here, an RFID tag is attached in home user's ID.

As shown in Figure 3, proposed service architecture is consist of RFID system and wireless sensor network for home user identification and context sensing.

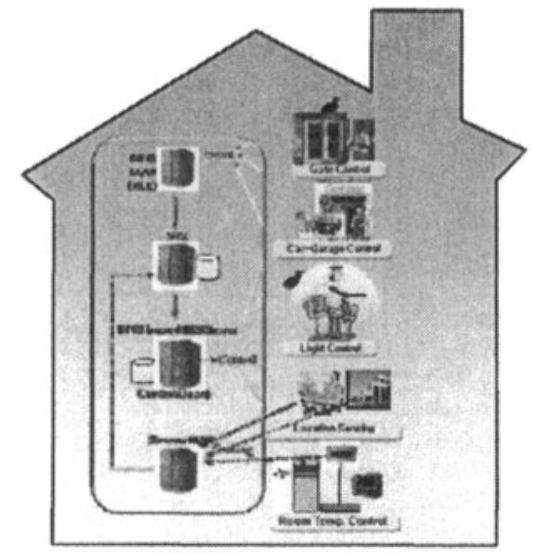

Figure 3. The service architecture of $\mathrm{u}$-IHS system.

Our system for intelligent home networking management service[6] is consists of following elements.

- RFID Tag, IP based Sensor and Sensor networks

Capturing : Home User, Car, etc.

. Sensing : User's motion, Location, Temperature, Humidity, Luminance, etc

- RFID Home Reader,

- mobile phone with RFID Reader

- RFID Home Middleware

- Sensor Home Middleware

- Secure IS server for RFID and sensor

- Context aware IHS Server

- Security and Privacy management by dynamic context policy 
. Real-time SMS Notification : Safety situation to user

. Controlling : Heating Device, Air conditioning

- Device, Lightings, Locking Device of Door, Gate of cargo, TV, window, curtain and safety level in home security system etc.

\section{Context aware dynamic reconfiguration of privacy and security for u-IHS service}

We considered a smart home scenario in which appliances in the kitchen can only be activated in the presence of an adult. And if the temperature at the stove is getting higher or the smoke alarm detects some amount of smoke, heat from the stove can be automatically turned off. Hence, instead of fixed security levels or security action, it is possible to map home user's context to security action, safety levels and safety related actions such as Figure 3.

Figure 3 depicts the classified context based RFID and WSN service architecture which is based on functions for supporting user's situation awareness concept and related operations. There are the two sides from the home service access view points. One is the local service access at inside home or home area network. The other is the outside of home. It will be remote service access from the customer attached to the $\mathrm{BcN}$ networks

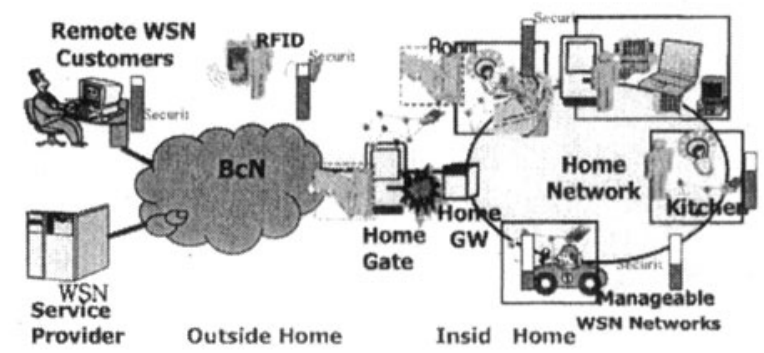

Figure 3. Dynamic Security Management with context aware mechanism

The default privacy protection level is determined based on the privacy impact assessment result undertaken by the government and the default privacy protection level is stored as the default policy of the tag and the home server. And user also can manage the privacy level by subscription and modification, after he purchases the product. 
We begin by describe the network configuration and system features for u-IHS in this section. A context based processing system is designed to safe home environment, and it is described in Figure 4.

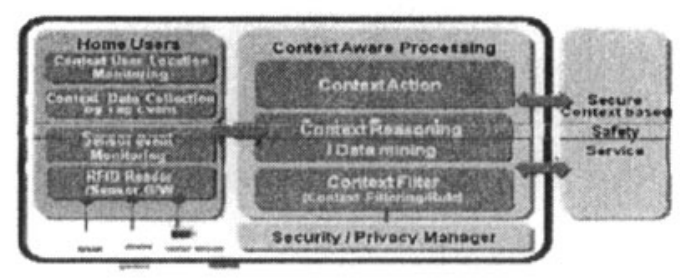

Figure 4. The block Structure of a context aware safety Management

In this model, the home user holding the RFID tagged identification card create capturing event and location event by reader and location sensor in door, kitchen or room and the event data and sensing data is transfer to back-end information server via middle-ware. The information server subsequently finds the matching context rule ID and selects context service server. Specifically, we consider a safety and context aware scenario in u-IHS system where. The secure information server sends the received RFID event and sensor data and parameters to IHS system. Then, IHS verifies the family or personal context policy for authorization and transform into each control board or control system for context based identity or safety and sophisticated control.

In view of information security protection, a serious problem for the RFID and sensor network service is a threat to DoS and information hacking. Here, the safety issue involves the risk of exposing information stored in IHS or other server and the leakage of information including personal moved data related to the user's home. Verification in IHS is for checking the user's context based safety level and user's context based privacy policy. Whenever RFID events and continuous sensing context value is received in secure IS server, it is transfer to meaningful context data and events, the IHS server checks the filtered and selected information and authorize user's context based safety level.

Our model of context based home networking safety and system in this regard, ID and parameter encryption and access control are used for prevention of service hacking or DoS attack in data transportation[6]. Integrating security function and intelligent context based safety and control mechanism in home network is particularly challenging due to the high demands made on their dependability[10][11].

The IHS server control machines and equipments in room automatically by event. The typical equipments of home are gate, parking garage, air conditioner, 
heater, TV, audio, window, curtain and all other home device for user's convenience by notified web service.

\section{System Design of Combined System with Context aware and Dynamic Security mechanism}

In this section we discuss the functions and block diagram for the RFID sensor network based context aware system, which provides a automatic and secure controlling service by capturing the tag(sensor) and get out of the user's information.

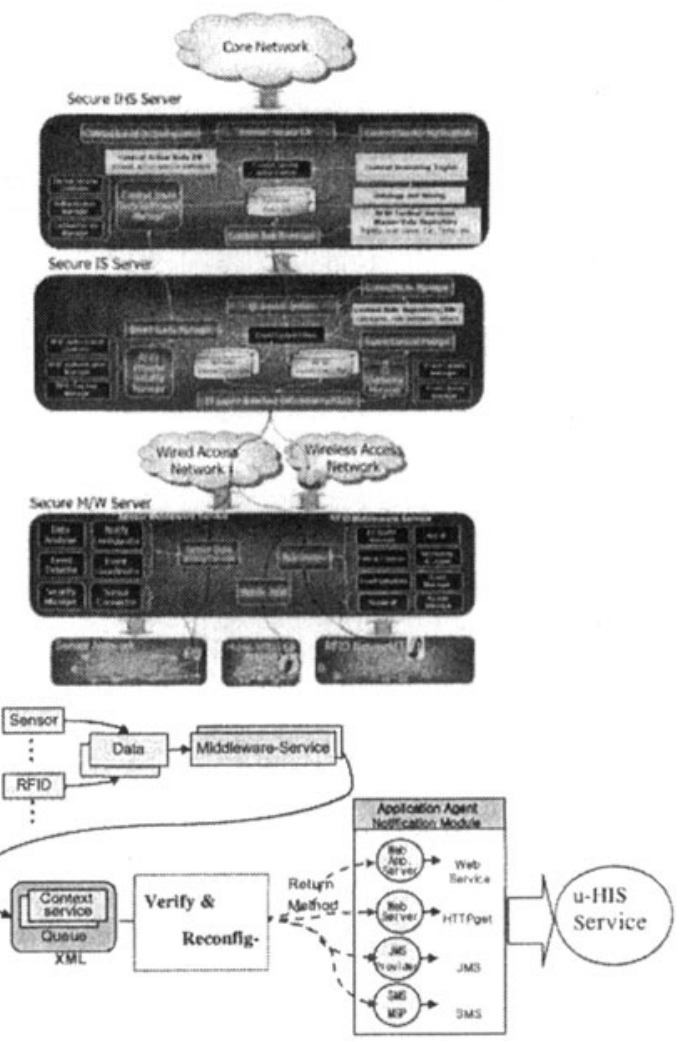

Figure 5. The proposed system design for context-aware home safety and managed security control service 
First, our IS server architecture is context driven in nature, defined context events are connected to specific service as a continuous tree topology, as shown in Figure 5. The Secure IS Server in Figure 5 represents the information server and context processing point, event data flow context filter and mapped by context rules in a continuous event unit. Data processing which needs continuous monitoring can be performed. The processed result is collected in result queue for interworking with application. These results are connected to proper application system by return methods. The return methods are Web service, HTTPget, JMS and mobile phone SMS for user's urgent event. This approach is desirable when home intelligent system, such as IHS need to be connected IS server with different way and different control network system. A variety of Web servers for context aware service and control are available. The main advantage of this approach is the secure connection and context policy based home device control and safety management. In particular, WS security and AS2 binding is used for secure transmission between servers. The IHS server is connected to IS server for family or personal context based service, covered in[7]. For context processing, the IHS server stored user's context policy data. Protocol and context rule and language like XML based GPDL(Generalized Policy Definition Language) are designed for context policy management. Each policy contains a subject role(the subject on which the policy is to apply), and object role(the object to which the policy applies) and action(on the object), an environment role(the context of the action) and the permissions(whether the action is to be permitted or not).

Context policy based detailed safety and access control mechanism is desired, since someone request and receive the context information of specified person without approval. Figure 3 describes the context based flow diagram for supporting user's situation awareness concept and related security operations.

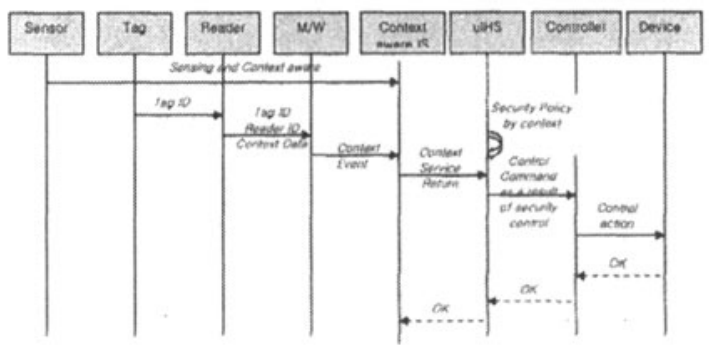

Figure 5. The proposed flow design of context-aware security control

The security level of context data defines different level for user's situation and context. And he wants to make the decision whether provide or not. The Information server stores the context result data and event logs by context policy and transfers them to the IHS server. The user can also re-configure the context action 
preference in IHS. From this point of view, we define access control features as following way.

. Access control of the context result data of an RFID/sensor

. Access control of the source data of an RFID/sensor

. Access right of a specified log data

. Mode of operator

(General Administer/Part administer/User etc)

The Secure M/W Server is consists of two part, one is for wireless sensor network and the other is for RFID middle-ware service. In this section, we discuss the IHS application server aspects of our system. For automatic control service, we designed IHS server, device control board and control software. The advantages of this system are as follows: first, we provide context based security management in home safety management aspects. Second, energy efficiency in home maintenance can be achieved. Third, advanced automatic home conditions are supported by utilizing the identity and context awareness in u-IHS system.

\section{Conclusion}

In this paper, we considered intelligent home safety management service by using context aware u-IHS system.

We designed the secure and automatic control service using RFID tagged ID card and context based light and temperature management using sensor and RFID tag. And we also describe the automatic parking garage control service using RFID tagged car. In the proposed u-IHS system, we presented in terms of context policy whereas we introduced a context based action scheme using configured context authorization in IHS. By doing so, context based dynamic security/privacy management and also intelligent home safety can be realized.

In this regard, the suggested u-IHS system is an effective solution for contextaware home safety management service using RFID and Sensor network.

\section{Acknowledgements}

This work was supported by the IT R\&D program of MIC'IITA. [2005-S088-03, Development of Security technology for Secure RFID/USN Service]. 


\section{References}

[1] Siio, I., Hayasaka, T., "Putting information to things : RFID tags and their application", IPSJ Magazine, Vol. 40, No.8, pp. 846-850, Aug. 1999.

[2] EPCglobal. "The EPCglobal Network:Overview of Design, Benefits and Security", http://www.epcglobalinc.org/Sep. 2004.

[3] G. Avoine and Ph. Oechslin, "A scalable and provable secure hash based RFID protocol", International Workshop on Pervasive Computing and Comunications Security", PerSec 2005, pp. 110-114. IEEE, 2005.

[4] Sanjay E. Sarma, et al., "RFID Systems and Security and Privacy Implications", CHES 2002, Lecture Notes in Computer Science 2523, pp. 454-469. 2002.

[5] EPCglobla Inc. http://www.epcglobalinc.org/.

[6] B.G. Lee, H.W. Kim, "A Design of Privacy Conscious RFID System Using Customizing Privacy Policy Based Access Control", Lecture Notes in Computer Science Vol. 3823, pp. 673-682, Nov. 2005.

[7] G.D. Abowd, M. Ebling, G. Hunt, H. Lei, and H.W. Gellersen, "Context-Aware Computing," PERVASIVE computing. JULY-SEPTEMBER 2002, pp. 22-23.

[8] Chemishkian, S., "Building smart services for smart home", Networked Appliances, p.p 215-224, January 2002

[9] Jiang, L., Liu, D., Yang, B., "SMART HOME RESEARCH", Machine Learning and Cybernetics, p.p 659-663 vol. 2, August 2004

[10] Mostefaoui, G.K.., "Context-Aware Computing: A Guide for the Pervasive Computing Community", Pervasive Services, p.p 39-48, July 2004

[11] Wullems, C., Looi, M., Clark, A., "Towards context-aware security: an authorization architecture for intranet environments", Pervasive Computing and Communications, p.p 132-137, March 2004.

[12] Covington, M. J., Fogia, P., Zhiyuan, Z., Ahamad, M., "A Context-Aware Security Architecture for Emerging Applications", Annual Computer Security Applications Conference, p.p 249-258, December 2002. 